\title{
Putting Fine Particulate Matter and Dementia in the Wider Context of Noncommunicable Disease: Where are We Now and What Should We Do Next: A Systematic Review
}

\author{
Ruth Peters $^{\mathrm{a}, \mathrm{b}}$ Ian Mudway ${ }^{c}$ Andrew Booth $^{d}$ Jean Peters ${ }^{\mathrm{d}}$ Kaarin J. Anstey ${ }^{\mathrm{a}, \mathrm{b}}$ \\ aSchool of Psychology, University of New South Wales, Sydney, NSW, Australia; beuroscience Research Australia, \\ Sydney, NSW, Australia; 'MRC-PHE Centre for Environment and Health, NIHR Health Protection Research Unit in \\ Environmental Exposures and Health, School of Public Health, Imperial College London, London, UK; dSchool of \\ Health and Related Research, University of Sheffield, Sheffield, UK
}

\section{Keywords}

Air pollution - Dementia - Cognitive impairment .

Particulate matter - Noncommunicable disease

\begin{abstract}
Introduction: A significant proportion of the global population regularly experience air quality poorer than that recommended by the World Health Organization. Air pollution, especially fine particulate matter $\left(\mathrm{PM}_{2.5}\right)$, is a risk factor for various noncommunicable diseases (NCDs) and is emerging as a risk factor for dementia. To begin to understand the full impact of $\mathrm{PM}_{2.5}$, we review the longitudinal epidemiological evidence linking $\mathrm{PM}_{2.5}$ to both dementia and to other leading NCDs and highlight the evidence gaps. Our objective was to systematically review the current epidemiological evidence for $\mathrm{PM}_{2.5}$ as a risk factor for cognitive decline and incident dementia and to put this in context with a systematic overview of $\mathrm{PM}_{2.5}$ as a potential risk factor in other leading NCDs. Methods: We performed 2 systematic reviews. A highlevel review of reviews examining the relationship between $\mathrm{PM}_{2.5}$ and leading NCDs and an in-depth review of the longitudinal epidemiological data examining relationships between $\mathrm{PM}_{2.5}$ incident dementia and cognitive decline. $\boldsymbol{R e}$ -
\end{abstract}

sults: There were robust associations between $\mathrm{PM}_{2.5}$ and NCDs although in some cases the evidence was concentrated on short rather than longer term exposure. For those articles reporting on incident dementia, all reported on longer term exposure and 5 of the 7 eligible articles found $\mathrm{PM}_{2.5}$ to be associated with increased risk. Conclusion: The evidence base for $\mathrm{PM}_{2.5}$ as a risk factor for dementia is growing. It is not yet as strong as that for other NCDs. However, varied measurement/methodology hampers clarity across the field. We propose next steps.

(c) 2021 S. Karger AG, Basel

\section{Introduction}

In recent years, the established literature linking poor air quality to adverse cardiopulmonary end points, including excess mortality [1], has expanded to include evidence of associations with incident dementia [2-4]. In 2018, we reviewed the evidence for air pollution and cognitive decline or dementia and noted that these adverse associations appear strongest when considering longer term exposures to ambient fine particulate matter $\left(\mathrm{PM}_{2.5}\right)$. $\mathrm{PM}_{2.5}$ refers to particles with an average aerodynamic di- karger@karger.com

(c) 2021 S. Karger AG, Basel

www.karger.com/ned

Karger ${ }^{\prime}$ -
Correspondence to:

Ruth Peters,r.peters@neura.edu.au 
ameter of $\leq 2.5 \mu \mathrm{m}$ and is composed of a mixture of chemical components, primary emissions, and products of secondary chemical reactions in the atmosphere, derived from a wide range of sources $[5,6]$. The $\mathrm{PM}_{2.5}$ ambient mass concentration, averaged over varying lengths of time, reflects the sum of all these sources and provides a single uniform metric to interrogate population health, often based on modeled attributions, but ultimately informed by widespread monitoring of this pollutant for regulatory and research purposes. Globally, the World Health Organization (WHO) estimates that around 91\% of the population live in places where annual fine particulate matter $\left(\mathrm{PM}_{2.5}\right)$ levels regularly exceed the recommended WHO guideline level of $10 \mu \mathrm{g} / \mathrm{m}^{3}$, with those in low- and middle-income countries most at risk [3].

Importantly, there are also established links between leading noncommunicable diseases (NCDs) (e.g., diabetes and stroke) and an increased risk of later dementia [5]. This is important since $\mathrm{PM}_{2.5}$ may raise the risk of dementia via both direct and indirect pathways. For example, dementia risk may be increased via inflammatory respiratory disease pathways and/or cerebrovascular disease [6, 7]. Given the potential overlap between $\mathrm{PM}_{2.5}$ as a risk factor for NCDs and a fast emerging risk factor for dementia, it is important to understand its population-level impact. Here we draw together the epidemiological evidence linking $\mathrm{PM}_{2.5}$ exposure to incident leading NCDs (using review of review methodology) and also systematically review the longitudinal epidemiological evidence on the relationship between $\mathrm{PM}_{2.5}$ and incident dementia and cognitive decline. We present the extent and scope of the evidence to date, highlighting the gaps and proposing the next steps.

\section{Methods}

Standard systematic review methodology [8] was used to undertake 2 complementary literature searches. The first was a highlevel systematic update focused on $\mathrm{PM}_{2.5}$ as a risk factor for leading NCDs followed by an in-depth systematic review examining the relationship between $\mathrm{PM}_{2.5}$ and incident cognitive decline and dementia. For both reviews, there were 2 independent analysts (R.P. and J.P.). The lead analyst carried out the literature searches. All identified abstracts, or titles where abstracts were unavailable, were double reviewed and a list of potentially relevant references compiled independently by the 2 analysts. These lists were compared, and differences were resolved by discussion. Once the list of possible references was agreed, full-text articles were obtained, independently read, and assessed for relevance. Data were extracted by the lead analyst and checked by the 2nd analyst. Standard extraction tables were used.
Major Noncommunicable Diseases and $\mathrm{PM}_{2.5}$

Leading NCDs were defined as cardiovascular disease (myocardial infarction, heart failure, and stroke), respiratory disease (COPD and asthma), lung cancer, diabetes mellitus, or chronic kidney disease (CKD), based on the WHO top 10 NCD causes of death [9]. To evaluate the relationship between exposure to $\mathrm{PM}_{2.5}$ and leading NCDs, the databases MEDLINE, Embase, and PsycInfo ${ }^{\circledR}$ were searched from inception to January 31,2020 . A review of reviews methodology was selected as a systematic method frequently used to summarize large volumes of data from an established literature and to ensure inclusion of most comprehensive and recent evidence $[10,11]$. Search terms included (air pollut* or particulate or $\mathrm{PM}_{10}$ or $\mathrm{PM}_{25}$ or Roadway or Vehicle or Diesel.ti.) and (systematic review.ti or systematic.af) (see online suppl. Text 1 ; for all online suppl. material, see www.karger.com/doi/ $10.1159 / 000515394)$. Utilizing methodologies adapted from those that underpin guideline development [12], we included only the most recent systematic review reporting on incident or worsening $\mathrm{NCD}$ and exposure to $\mathrm{PM}_{2.5}$. We did not include cross-sectional relationships, diagnoses that may predispose to NCDs (e.g., insulin resistance, hypertension, and obesity), and data from child or adolescent populations, those reporting composite outcomes or solely fatal outcomes. Data were extracted from the systematic reviews on the number of constituent studies, the regions of the world where the studies had taken place, the way the exposure had been assessed, the $\mathrm{PM}_{2.5}$ exposure estimates for the population, the assessment of incident disease, and the results. The Assessing the Methodological Quality of Systematic reviews (AMSTAR) version 2 (https://amstar.ca/Amstar-2.php) was used to evaluate the systematic reviews [13].

\section{Dementia, Cognitive Decline, and $P M_{2.5}$}

To evaluate the relationship between exposure to $\mathrm{PM}_{2.5}$ and incident cognitive decline or dementia (including incident Alzheimer's Disease), the databases MEDLINE, Embase, and PsycInfo ${ }^{\circledR}$ were searched from 2018 to April 1, 2020, supplemented by a prior search from inception to September 2018 [14]. Search terms included (alzheime* or dementia or cogniti*) and (air pollut* or particulate matter or roadway or particle size or $\mathrm{PM}^{*}$ or vehicle or diesel) (online suppl. text 1 ). The results were further strengthened using forward citation searching for each of the included articles published prior to September 2018 and examining each cited article against the inclusion and exclusion criteria. Articles were included if they reported on longitudinal studies evaluating the relationship between exposure to outdoor $\mathrm{PM}_{2.5}$ and incident cognitive decline or dementia, in human adults aged 18 years and older. Studies reporting indoor exposure or examining passive smoking were excluded. Where more than one article reported on the same population, the article including the largest number of participants was included.

For the relationship between $\mathrm{PM}_{2.5}$ and incident cognitive decline or dementia (including Alzheimer's Disease), information was collected on the dates of exposure, duration of follow-up, assessment of incident cognitive decline or dementia, $\mathrm{PM}_{2.5}$ concentrations, region of the world where the studies had taken place, and the results. Where multiple results were available, the most conservative interpretation, that is, selecting the longest exposure and most adjusted model, was reported. Data were extracted on length, dates and measures of exposure, region of recruitment, number of participants and participant age, average $\mathrm{PM}_{2.5}$ level, assessment of 


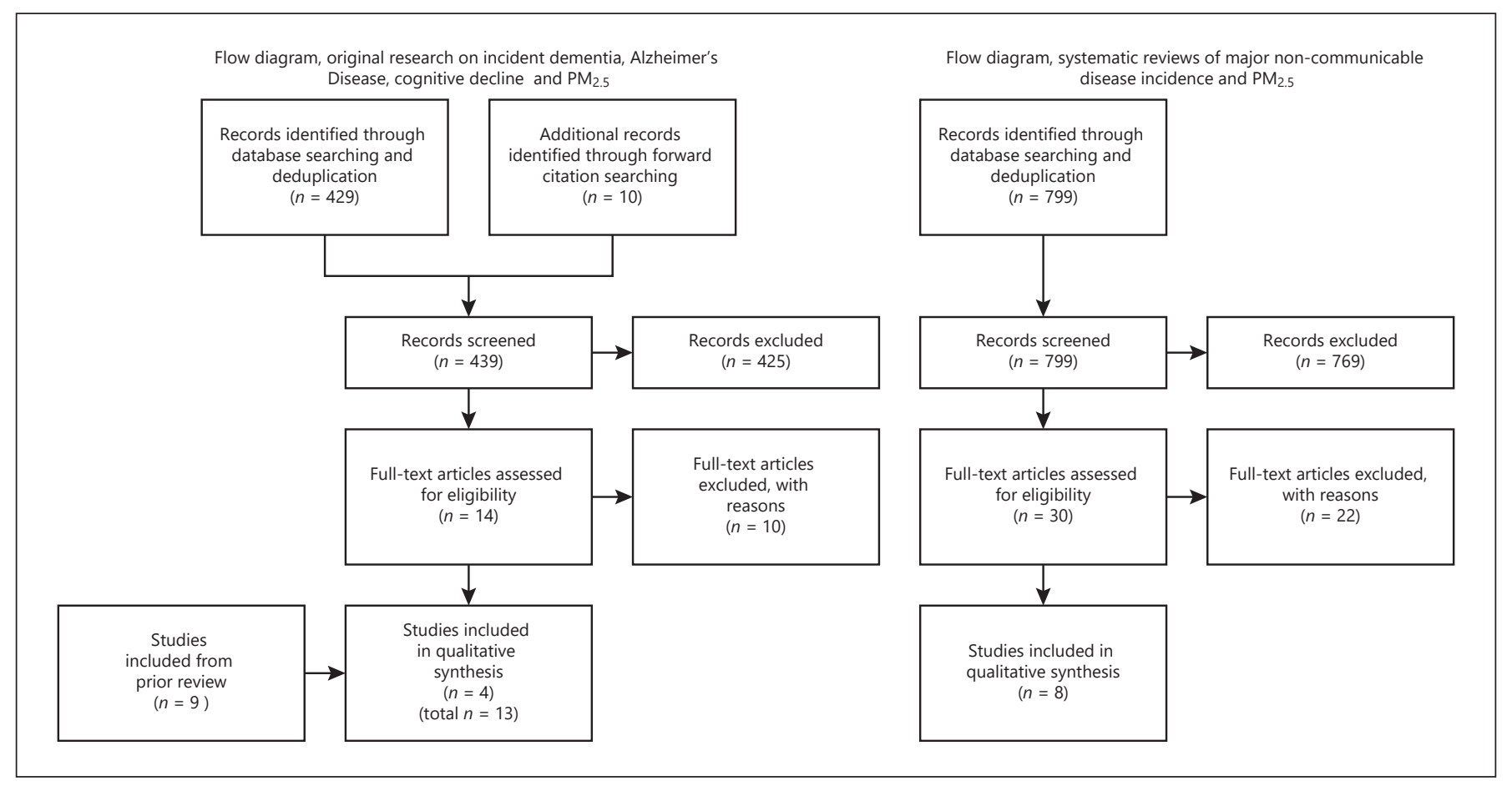

Fig. 1. Flow diagrams detailing the screening and inclusion processes.

dementia or cognitive decline, results, and evaluation of potential confounders. Each original research article was also assessed for bias against key criteria based on the Critical Appraisal Skills Programme (CASP $\odot$ ) cohort study checklist [15], and potential sources of bias in each study were tabulated.

\section{Results}

\section{Major Noncommunicable Disease and $P M_{2.5}$}

For the searches of systematic reviews reporting on the relationship between exposure to $\mathrm{PM}_{2.5}$ and incident NCD, 799 abstracts were screened and 30 assessed at the full-text stage. Twenty-two were excluded after full-text screening. Sixteen of these reviews had been superseded by more recent reviews, 2 were of the same year as alternative reviews, but more limited. In one review, it was not possible to separate out the results for adults and children, one did not separate out $\mathrm{PM}_{2.5}$, one did not exclude crosssectional data, and one focused on methodology (online suppl. Table 1). No reviews were excluded based on their assessment of exposure (duration or methodology). Figure 1 for a flow chart. Reviews included case control, crossover, and time series studies with 2 [16] to 59 [17] studies included in meta-analyses.

Evidence on PM2.5, Dementia, and Noncommunicable Disease
Systematic reviews were identified that reported on the association between $\mathrm{PM}_{2.5}$ and incident or exacerbated respiratory disease (lung cancer [18] chronic obstructive airways disease [COPD] [17], asthma [19]), diabetes mellitus (DM) [20], cardiovascular disease (heart failure [21], stroke [22], myocardial infarction [MI] [23]), and chronic kidney disease (CKD) [16] (Table 1). Follow-up was both long term (3-34 years for the constituent studies on lung cancer [18]), 1-10 years for DM [20], 2 years for CKD [16], and short term with acute exposures of 0-3 days for COPD [17] and 0-7 days for stroke, heart failure, MI, and asthma [19, 21-23]. All meta-analyses reported a statistically significant relationship between their respective outcome and $10 \mu \mathrm{g} /$ $\mathrm{m}^{3}$ increment in $\mathrm{PM}_{2.5}$ with most point estimates falling between 1 and 10\% increase in risk. See Table 1 (in brief, estimated percentage increase and $95 \%$ confidence intervals from meta-analyses, for lung cancer: 9 (4-14), COPD: 2 (1-4), asthma: 3 (1-5), DM: 10 (4-17), heart failure: 112 (42-182), MI 2 (2-3), and stroke $1(1-1)$ ).

\section{Dementia, Cognitive Decline, and $P M_{2.5}$}

For the searches relating to the relationship between exposure to $\mathrm{PM}_{2.5}$ and incident cognitive decline or dementia (2018-present), 439 abstracts were screened and 14 assessed at the full-text stage. Ten were excluded after 


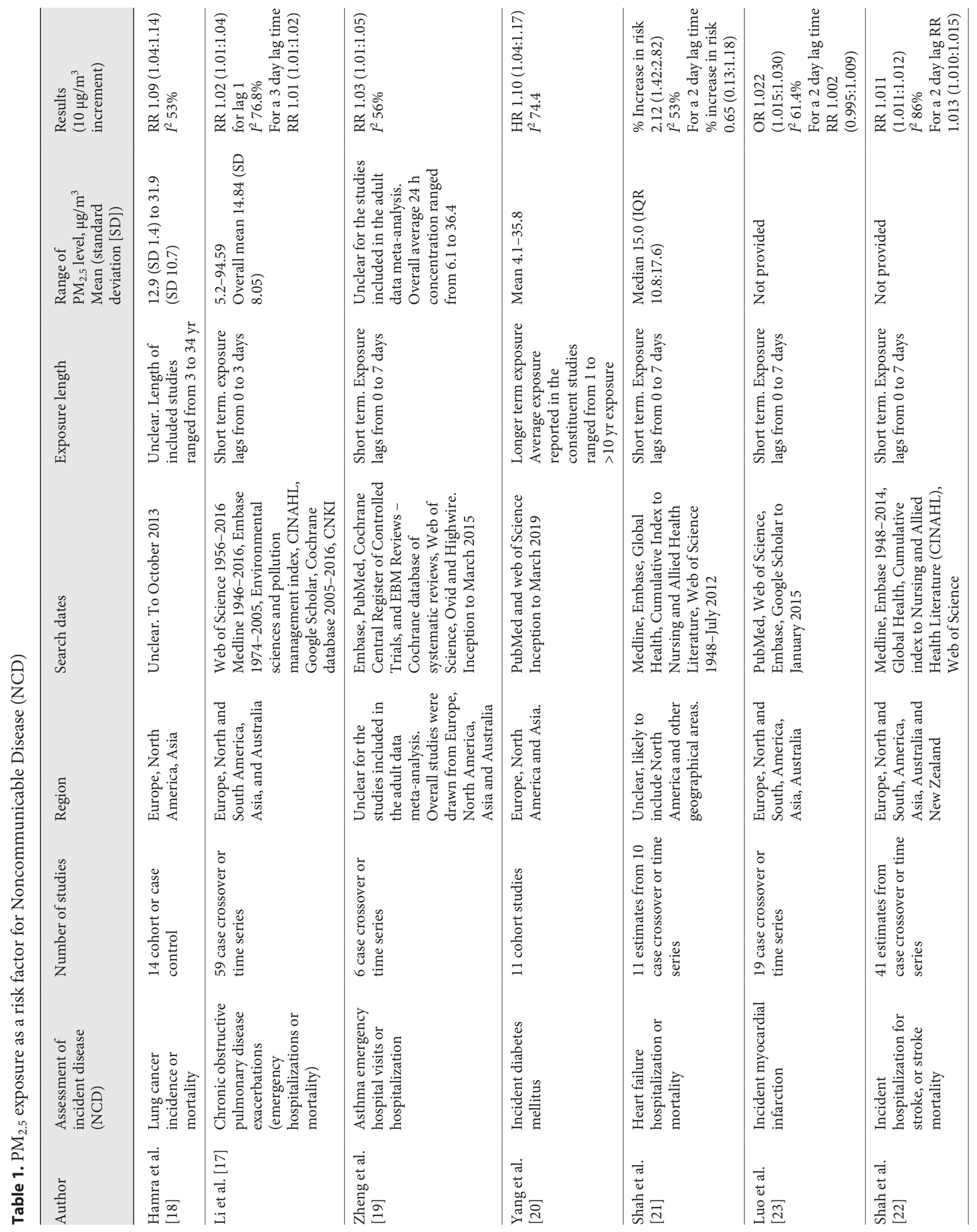




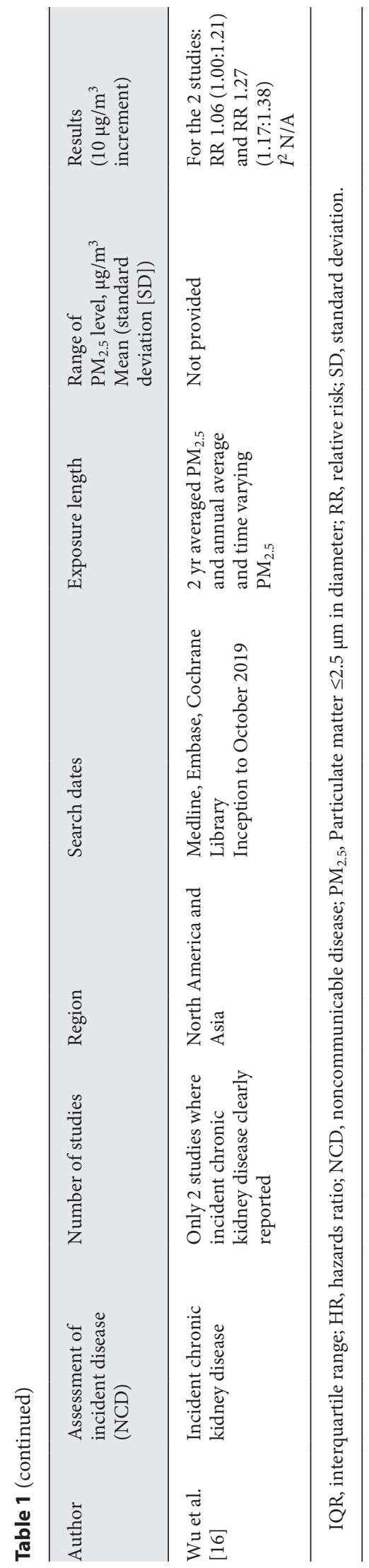

Evidence on PM2.5, Dementia, and Noncommunicable Disease full-text screening (online suppl. Table 2), one did not report results for PM2.5, three either included prevalent dementia, or it was not possible to tell whether it was excluded, one reported only fatal outcomes, 3 appeared to report on subpopulations of studies already included, and 2 reported cross temporal analyses without measuring incident decline. Four articles were included ([24-27]), and these were supplemented with 9 articles that had been identified in our prior systematic review (covering the literature from inception to 2018) but which also met the inclusion criteria [28-36], Tables 2 and 3, and Figure 1 for the flow chart.

Seven studies reported on incident dementia $[25,27$, 31-34, 36] (Table 2), 5 of the 7 studies reporting on incident dementia used administrative health records for case ascertainment, selecting out coded incident dementia based on the International Classification of Disease codes versions 9 or $10([27,31,33,34]$, read codes (used in UK general practice) [34], and/or the Diagnostic Statistical Manual version 4 (DSM-IV) $[31,36]$. The 3 smallest studies were research cohorts. These included (i) the Betula cohort from Sweden $(n=1,806)$ [36], which reported additional review by old age psychiatrist, for dementia diagnosis, (ii) the Swedish National Study on Ageing and Care in Kungsholmen (SNAC-K) $(n=2,927)$, which used physician review [25], and (iii) the Cacciolotto et al. [32] study population $(n=3,647)$, which was drawn from the larger Women's Health Initiative Memory (WHIMS) Study. WHIMS was a research-based cohort with repeated assessments that diagnosed dementia with a multistep process using the extended Mini-Mental State Examination (MMSE) for screening. Those that screened positive received further neuropsychological and physician assessment plus imaging [32]. The studies that used health records were population based and reported on between 100,000 and over 2 million individuals [27, 31, 33, 34]. Although age was not consistently reported, the mean baseline age for all dementia studies was estimated at around 65 years. Exposure duration ranged from 1 [34] to 14 years [33] with $\mathrm{PM}_{2.5}$ data collected at different time points from 1990 [36] to 2012 [33]. Five of the 7 studies reported a relationship between exposure to $\mathrm{PM}_{2.5}$ and increased risk of incident dementia, but methodology varied between studies. Exposure was based around residential location, with several studies specifying details such as the use of postcodes or zip codes $[31,33]$ taking account of the history as well at the present residential location [32], or use of grids as small as $50 \times 50 \mathrm{~m}$ in urban areas and 3,200 ×3,200 $\mathrm{m}$ in rural areas [36]. Annual exposure measures were most common [32-34, 36], but 


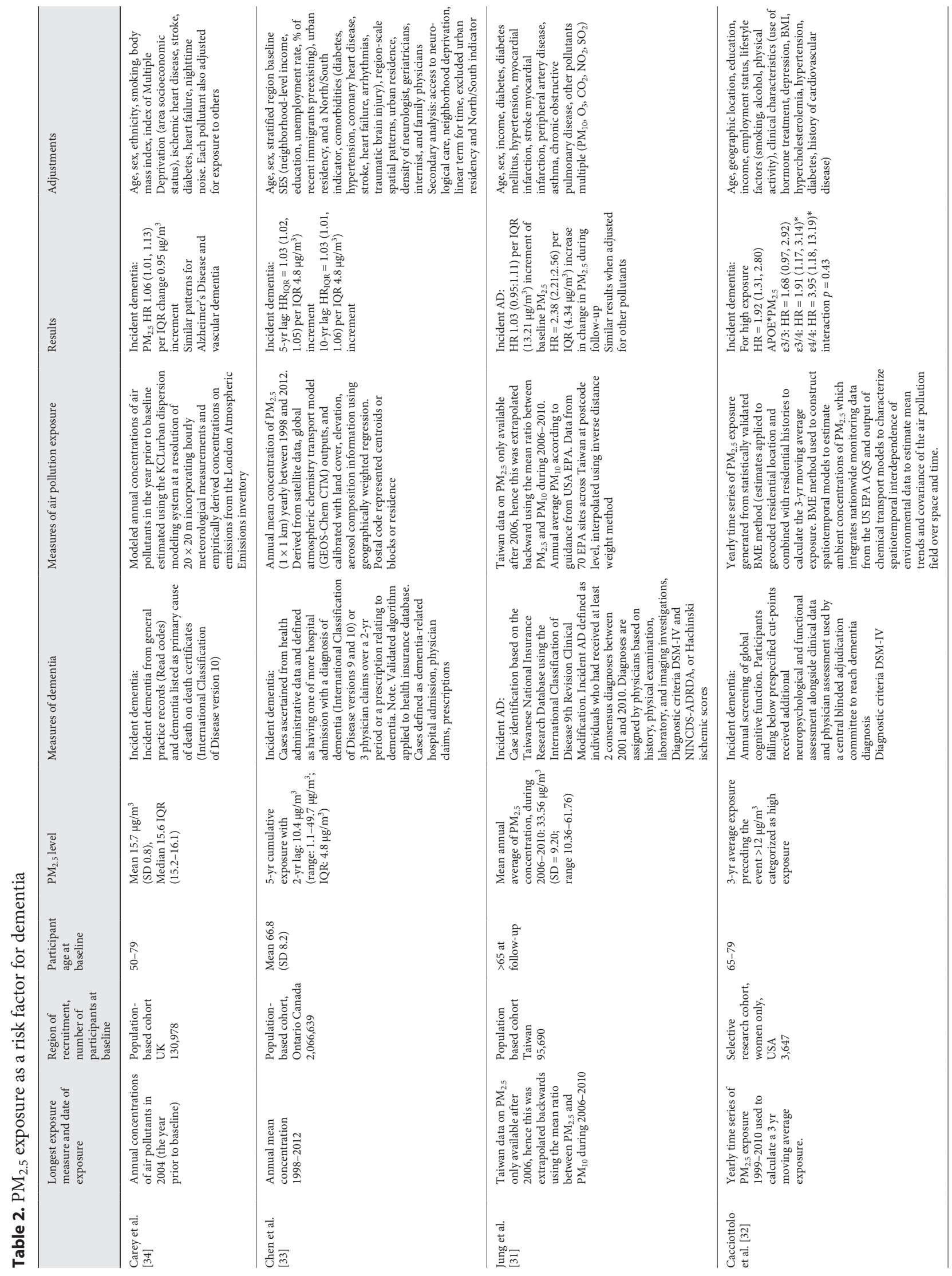




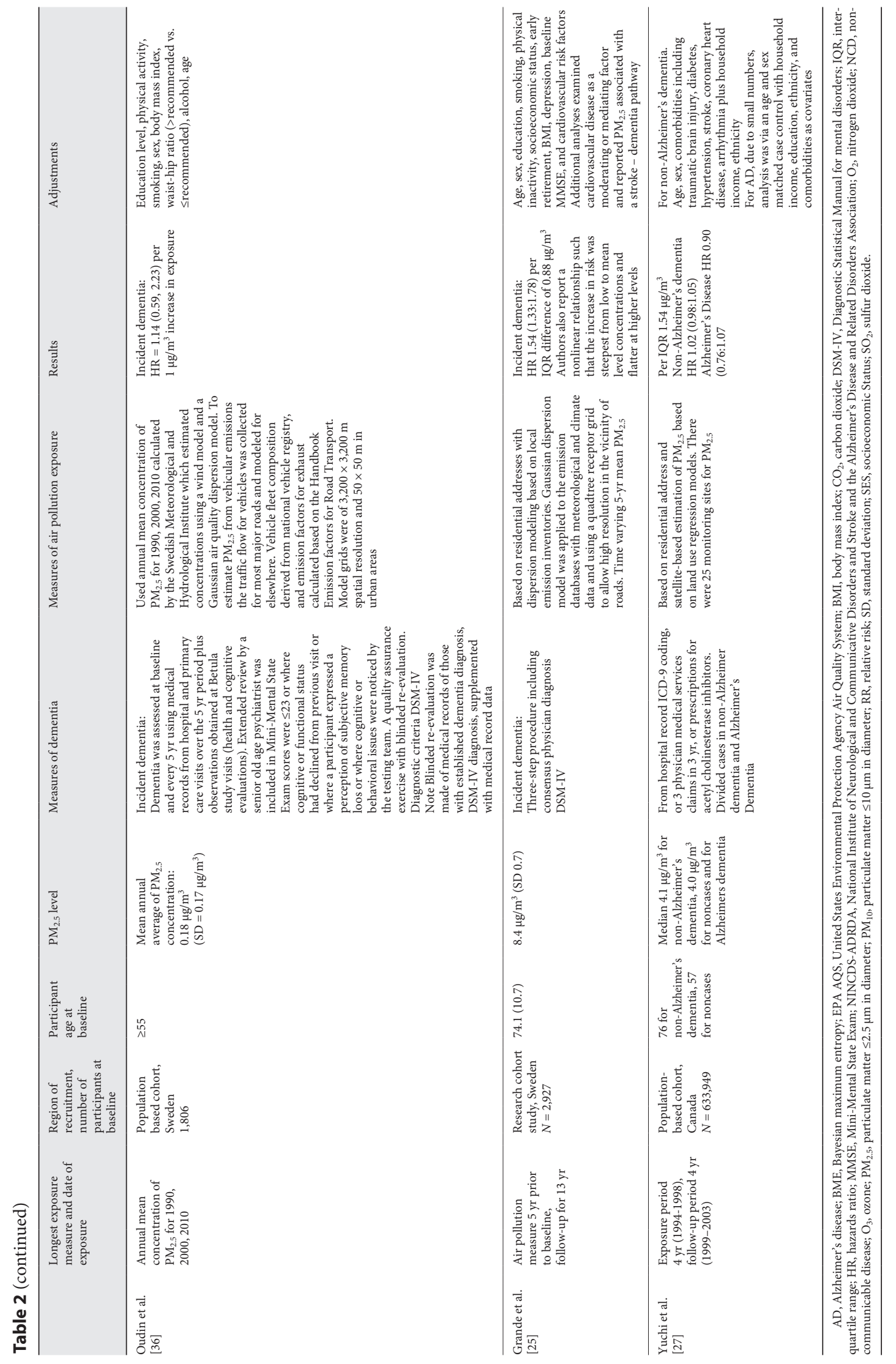




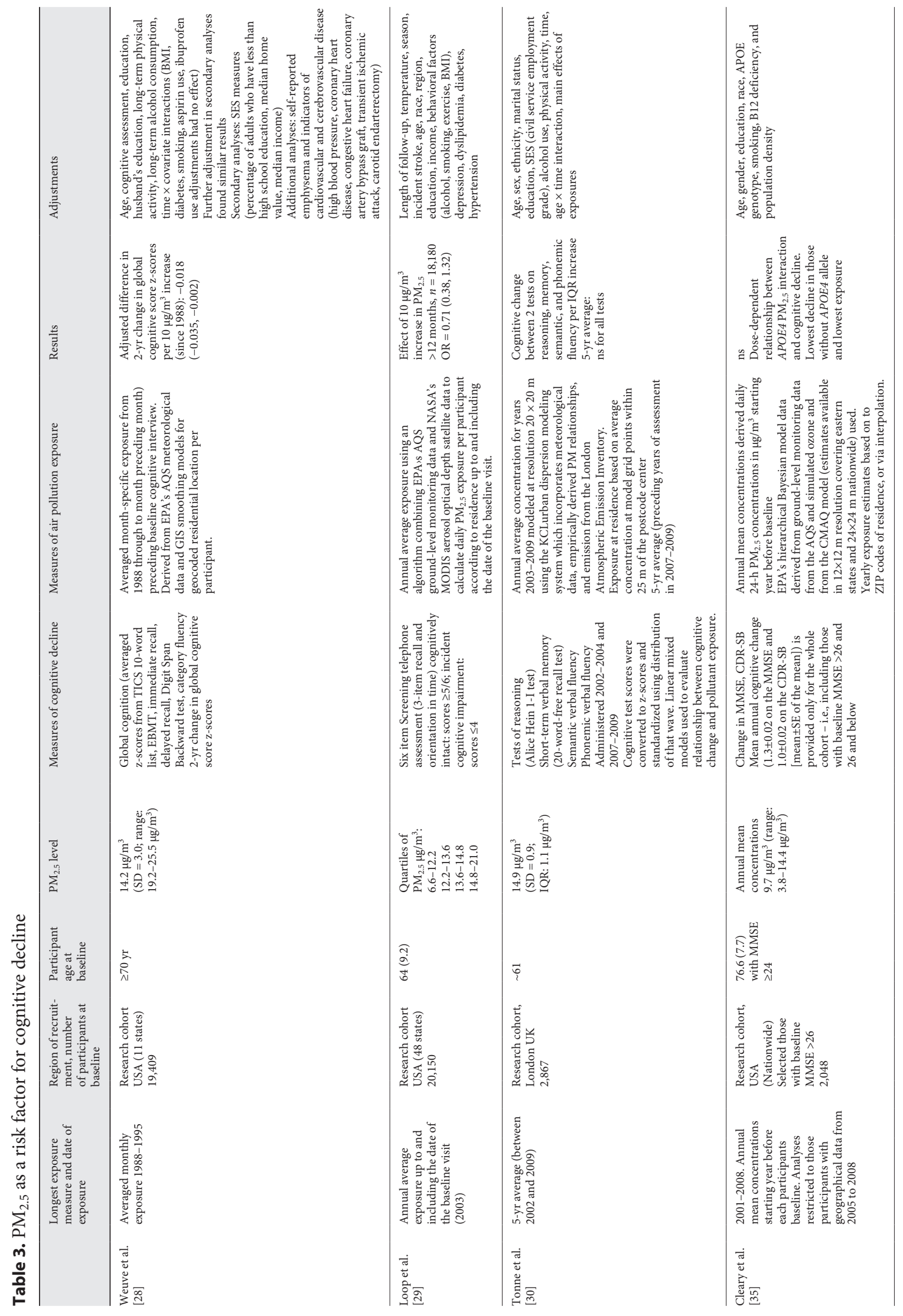




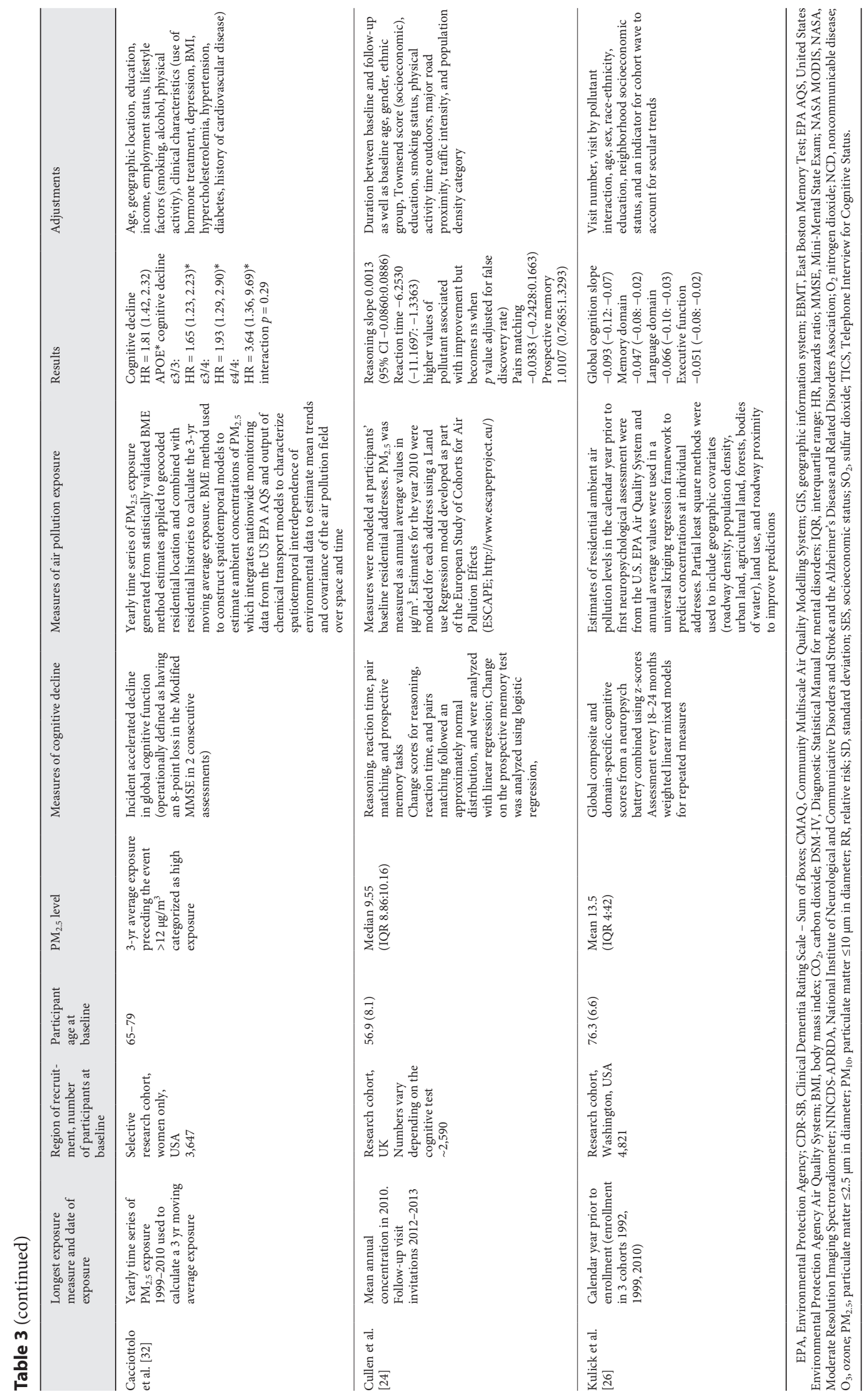

Evidence on PM2.5, Dementia, and 
exact measures, timing, and calculation of exposure variables differed. The USA study [32] reported a 92\% \{hazard ratio (HR) 1.92 (95\% confidence interval [CI]) 1.31:2.80\} increased risk for exposure to levels above 12 $\mu \mathrm{g} / \mathrm{m}^{3}$ over 3 years, the Canadian study reported a $3 \%$ (HR 1.03 [1.02:1.05]) increased risk with an increment of $4.8 \mu \mathrm{g} / \mathrm{m}^{3}$ [33], the UK study a $6 \%$ (HR 1.06 [1.01:1.13]) increased risk but for a $0.95 \mu \mathrm{g} / \mathrm{m}^{3}$ incremental change in exposure [34], and one Swedish study a 54\% (HR 1.54 [1.33:1.78]) increased risk per $0.88 \mu \mathrm{g} / \mathrm{m}^{3}$ increase [25]. The other studies found no increased risk including the Taiwanese study [31] which reported exposure measures of $33.6 \mu \mathrm{g} / \mathrm{m}^{3}$ and the study with the lowest concentrations, the Betula study reporting a mean annual average $\mathrm{PM}_{2.5}$ concentration of $0.18 \mu \mathrm{g} / \mathrm{m}^{3}$ (standard deviation [SD] $0.17 \mu \mathrm{g} / \mathrm{m}^{3}$ ) [36].

The 7 studies reporting on incident cognitive decline all comprised research cohorts (Table 2). Two were recruited from the UK $[24,30]$ and 5 from the USA $[26,28$, $29,32,35]$. Studies ranged in size from 2,048 [35] to over 20,000 [29] participants. Air pollution measures were collected as early as 1988 [28] and as recently as 2010 [24, 26, 32 ]. The mean age of the cohorts ranged from 56.9 (SD 8.1) [24] to 76.3 (SD 6.6) [26] years. All reported on cognitive change or incident decline defined as a fall to below a threshold [29] or of a certain size [32]. Some chose to report cognitive domains or general cognitive assessment and 3 used screening tools $[29,32,35]$. The relationships between air pollution and cognition were largely nonsignificant $[24,29,30,35]$. The exceptions were Weuve et al. [28] who reported a significant 2-year decrease in global cognitive z-score (per $10 \mu \mathrm{g} / \mathrm{m}^{3}$ increase in $\mathrm{PM}_{2.5}$ ) and the WHIMS cohort which found an 81\% (HR 1.81 [1.42:2.32]) increase in incident cognitive decline (an 8-point decline in extended Mini-Mental State Exam in 2 consecutive assessments) in the higher exposure group [32]. Levels of $\mathrm{PM}_{2.5}$ were similar across studies with mean concentrations of 14.9 [30] 14.2 [28], 13.6 (Median) [29], 13.5 [26], 9.7 [35], and $9.6[24] \mu \mathrm{g} / \mathrm{m}^{3}$.

\section{Assessment of Bias}

Overall, the risk of bias within studies reporting on the relationship between $\mathrm{PM}_{2.5}$ and incident dementia, or cognitive decline was low to moderate (6 rated as low, 6 as low moderate, and 1 as moderate) see online suppl. Table 3. Included studies reported clear aims, appropriate methodology, comprehensive adjustment for confounders, standard methods for measurement of exposure, and use of health records or appropriate cognitive testing for outcomes. Improvements within individual studies would involve improving detailed assessment of exposure, using more comprehensive neuropsychological testing and more rigorous case ascertainment, selecting more representative populations, and investigating attrition more thoroughly. For NCDs, the reviews were largely rated as moderate quality using the rigorous AMSTAR 2 criteria [13] (6 as moderate and 2 low quality).

\section{Across the Evidence Base}

A greater risk of bias becomes evident when the studies are narratively synthesized and considered together as a body of research [37]. For the studies on dementia and cognitive decline, although the evidence is largely from older adults, the differing lengths of exposure, methods of modeling of exposure and outcome, and varied combinations of confounders and potential under or over adjustment in the analyses (we selected the most adjusted results) make the studies appear less comparable than when assessed individually. For example, it is not possible to know whether the same positive or negative relationships would be shown across the studies if the same $\mathrm{PM}_{2.5}$ concentrations and exposure times had been used. Data are also drawn only from a limited number of high-income countries and are lacking for younger age-groups and longer term follow-up, particularly relevant for dementia where disease processes may start 10 or 20 years prior to symptom onset [38]. The reviews of other NCDs suffer from similar issues with some evidence only available for acute and very short-term exposure and some only for on longer term follow-up measures. Reviews demonstrate more global data than the studies of dementia, but most combine varied study designs and wide $\mathrm{PM}_{2.5}$ concentration ranges. Finally, for all review and original research studies, included in this systematic review, $\mathrm{PM}_{2.5}$ was defined based on its ambient mass concentration, which is insensitive to the significant regional differences in its composition [39-42].

\section{Discussion}

Our aim was to systematically review the evidence relating to $\mathrm{PM}_{2.5}$ and risk of dementia or cognitive decline, and to position this within a wider public health context. In addition, given that vascular disease itself is a risk factor for dementia [6,7], we also performed a systematic review of the evidence for $\mathrm{PM}_{2.5}$ and other relevant $\mathrm{NCDs}$, to contextualize these observations with other established diseases previously associated with $\mathrm{PM}_{2.5}$ exposures and sharing common underlying inflammatory pathways. 
Overall, for studies on cognition, we found that the evidence supporting an association between fine particulate matter exposures was strongest for dementia. Studies on cognitive decline were inconclusive, with only 2 of the 7 studies reporting an elevated risk both of which used general measures of cognition. Most of the studies on incident dementia demonstrated an increased risk with higher $\mathrm{PM}_{2.5}$ concentrations. Our results are broadly in agreement with prior reviews [4345]. The systematic review evidence for the other NCDs was clearer than the evidence for cognition and dementia; however, exposure times for some outcomes were effectively based on acute time periods, whereas others were over the longer term. Interestingly, the results for dementia outcomes were obtained despite most studies adjusting broadly for the presence of other cardiovascular comorbidities, which may support an independent $\mathrm{PM}_{2.5}$ dementia pathway. This potentially argues for a more direct linkage to Alzheimer's disease as opposed to vascular dementia, but this distinction needs further research beyond inference drawn from epidemiological observations.

Using the standard review methodology, we used published data and, in order to represent the evidence base on NCDs, selected a review of reviews method rather than reviewing primary research. Despite this, we undertook a rigorous systematic review approach and, while we can conclude that the emerging evidence base confirms that $\mathrm{PM}_{2.5}$ is associated with increased risk of dementia and other NCDs, consideration of the whole picture reveals several limitations.

Individual studies and reviews revealed no definitive sources of bias on formal assessment; however, when considered holistically the gaps in the evidence become clearer. We raise several issues that need to be addressed before using the evidence to inform policy. These limitations fall within 3 categories: (i) those that may be overcome in the shorter term, (ii) those that require additional research, and (iii) those that cannot be easily remedied without additional data collection. We discuss each of these and make recommendations below.

\section{Limitations that may be overcome in the shorter}

term. These include a lack of standardization in reporting and in analyses, varied adjustment for confounders, the potential for incomplete adjustment for confounders, including those that may influence lifestyle and health choices, a mixture of acute and longer term exposure estimates and a lack of enough accounting for attrition (particularly attrition due to other health conditions exacerbated by $\mathrm{PM}_{2.5}$ ).
Recommendations: The adoption of an agreed standardized data collection, processing, and analysis protocol and/or a 1 or 2 stage individual participant data meta-analysis (IPD-MA) to facilitate similar processing across studies. Examination of multiple NCD end points and analysis, taking account of competing end points and interactions is also needed, for example, looking at the direct and indirect pathways that may impact on dementia risk.

2. Limitations that require additional research: Variation in exposure attribution methods between studies, including an absence of detailed compositional information, despite clearly different source profiles between countries, and within countries over time. Because $\mathrm{PM}_{2.5}$ is not a uniform chemical entity, its composition varies markedly between different regions $[40,41]$ as well as across time [40, 41]. While the epidemiological literature strongly focuses on $\mathrm{PM}_{2.5}$, it is notable that preliminary work and evolving research hypotheses around the potential causal link between air pollution and dementia have focused on primary combustion and mechanical abrasion particles [39] which represent only a fraction of $\mathrm{PM}_{2.5}$ mass and display marked spatial variation, not captured by the simple mass metric. As these fractions of $\mathrm{PM}_{2.5}$ are not widely measured in regulatory networks and as models for these metrics are relatively recent [42], their association with dementia incidence has not been fully explored. This may be of particular importance for cognitive outcomes since the ultrafines (particles that are $<100 \mathrm{~nm}$ in diameter) within $\mathrm{PM}_{2.5}$ may translocate across biological barriers, for example, via olfactory neurons to the olfactory bulb. Often $\mathrm{NO}_{2}$, for which widespread monitoring and well validated models are available, is used as a proxy for primary exhaust emissions from diesel vehicles, but the literature linking $\mathrm{NO}_{2}$ to dementia, or indeed to associated cardiovascular risk factors remains equivocal [2, 14]. Furthermore, there remain significant gaps in our knowledge of the molecular triggers and causal pathways linking poor air quality to increased dementia risk. Recommendations: We need a better understanding of how $\mathrm{PM}_{2.5}$ composition has varied over time and presently varies between different global regions, to fully interrogate and integrate studies drawn from different periods and locations. A better understanding of these issues will help inform causal inference and potentially improve our understanding of the drivers for some of the heterogeneity observed in the current evidence base. In addition, the extent to which $\mathrm{PM}_{2.5}$ and its constituents interact with copollutant gases and volatile organic 
species in the atmosphere, either additively or synergistically, to impact on the risk of one or more NCDs and pre-NCD states need to be clarified. This is particularly important where one NCD may impact on the risk of another, an area where evidence is severely lacking, such as for the relationship between cardiovascular disease and dementia [25]. Alongside this, we need a greater background understanding of the shape of the relationship between $\mathrm{PM}_{2.5}$ and NCD outcomes and the role of other risk factors. For example, is the relationship linear or is there a point at which it has less of an impact (as implied by the recent Grande et al. [25] study)?

Furthermore, how does the background risk of the population, the life-course exposure, and population risk factor habits play a part? Relevant to this is a need to better assess personal exposure, to collect data from wider geographical and cultural areas, and to look by population subgroups where risk levels may vary. For example, women may be exposed to increased risk where they are the ones predominantly exposed to cooking on woodstoves, or men where they spend more time on busy streets outside the home.

3. Limitations that cannot be easily remedied without new data collection: There is bias inherent in the use of administrative health record data, a lack of sophisticated cognitive testing and an absence of long-term follow-up.

Recommendations: An IPD-MA may help in resolving these limitations with the use of $\mathrm{z}$-score change for cognition, but inevitably new studies are needed.

Overall, we can conclude it is likely that greater exposure to $\mathrm{PM}_{2.5}$ increases the risk of dementia, and that there is some evidence that this effect is independent of other cardiovascular comorbidities. It is also notable that the magnitude of the relationship between incident dementia and $\mathrm{PM}_{2.5}$ after adjustment for comorbidities is of a similar magnitude to that seen for cardiovascular disease. This would imply that the total health impact of ambient $\mathrm{PM}_{2.5}$ may have been significantly underestimated. Understanding the potential associations with dementia is therefore critical and whilst the evidence base is growing and strengthening, before we can take the next steps and calculate population attributable risk, estimate cost implications and model the effects of risk reduction, we need a more sophisticated analysis of the current evidence and, ideally, new data collection. Air pollution is pervasive and global. It holds numerous inter-related health implications. Using a systematic review process to integrate what is already in the literature has allowed us to synthesize findings across the breadth of evidence necessary to evaluate such a global

health phenomena and has allowed important commonalities across these fields to emerge. To develop our understanding of the relationship between air pollution and dementia, we must now look holistically beyond dementia.

\section{Acknowledgements}

The authors are supported by research grants from the National Health and Medical Research Council of Australia and the Australian Dementia Collaborative Research Centre.

\section{Conflict of Interest Statement}

The authors have no conflict of interest to report.

\section{Funding Sources}

ISM was part funded by the National Institute for Health Research Health Protection Research Unit (NIHR HPRU) in Environmental Exposures and Health at Imperial College in partnership with Public Health England (PHE). The views expressed are those of the author(s) and not necessarily those of the NHS, the NIHR, or the Department of Health and Social Care or Public Health England. K.J.A. is funded by ARC Fellowship FL190100011.

\section{Author Contributions}

Ruth Peters conceived the research, drafted the search terms, ran the searches, extracted the data, and drafted the article. Ian Mudway co-conceived the research, advised on the search terms, contributed to the drafting of the article and the critical interpretation of the results. Andrew Booth advised on the search terms, strategies and assessment of the evidence, and contributed to the drafting of the article and the critical interpretation of the results. Jean Peters co-conceived the research, double screened the articles, aided with extraction, and contributed to the drafting of the article and the critical interpretation of the results. Kaarin J. Anstey aided in the conceptualization of the article and contributed to the drafting of the article and the critical interpretation of the results.

References
1 U.S. EPA. Integrated Science Assessment (ISA) for Particulate Matter (Final Report, 2019).

2 Landrigan PJ, Fuller R, Acosta NJR, Adeyi O, Arnold R, Basu NN, et al. The Lancet Commission on pollution and health. Lancet. 2018;391(10119):462-512.

3 WHO. Ambient (outdoor) air pollution. World Health Organisation Fact Sheets; 2018.

4 Livingston G, Huntley J, Sommerlad A, Ames D, Ballard C, Banerjee S, et al. Dementia prevention, intervention, and care: 2020 report of the Lancet Commission. Lancet. 2020; 396(10248):413-46. 
5 Prince MAE, Guerchet M, Prina M. Dementia and risk reduction an analysis of protective and modifiable factors. Alzheimer's Disease International; 2014.

6 Peters R, Ee N, Peters J, Beckett N, Booth A, Rockwood K, et al. Common risk factors for major noncommunicable disease, a systematic overview of reviews and commentary: the implied potential for targeted risk reduction. Ther Adv Chronic Dis. 2019;10:2040622319880392.

7 Seaton A, Tran L, Chen R, Maynard RL, Whalley LJ. Pollution, particles, and dementia: a hypothetical causative pathway. Int J Environ Res Public Health. 2020 Jan 30;17(3).

8 Grant MJ, Booth A. A typology of reviews: an analysis of 14 review types and associated methodologies. Health Info Libr J. 2009 Jun; 26(2):91-108.

9 WHO. The top 10 causes of death. World Health Organization Fact Sheets. World Health Organization; 2018.

10 Smith V, Devane D, Begley CM, Clarke M. Methodology in conducting a systematic review of systematic reviews of healthcare interventions. BMC Med Res Methodol. $2011 \mathrm{Feb}$ 3;11(1):15.

11 Bellou V, Belbasis L, Tzoulaki I, Middleton LT, Ioannidis JP, Evangelou E. Systematic evaluation of the associations between environmental risk factors and dementia: an umbrella review of systematic reviews and meta-analyses. Alzheimers Dement. 2017 Apr;13(4):406-18.

12 WHO. Risk reduction of cognitive decline and dementia who guidelines. World Health Organization; 2019.

13 Shea BJ, Reeves BC, Wells G, Thuku M, Hamel C, Moran J, et al. AMSTAR 2: a critical appraisal tool for systematic reviews that include randomised or non-randomised studies of healthcare interventions, or both. BMJ. 2017;358:j4008.

14 Peters R, Ee N, Peters J, Booth A, Mudway I, Anstey KJ. Air pollution and dementia: a systematic review. J Alzheimers Dis. 2019;70(s1): S145-s63.

15 CASP. Critical Appraisal Skills Programme Checklists.

16 Wu MY, Lo WC, Chao CT, Wu MS, Chiang CK. Association between air pollutants and development of chronic kidney disease: a systematic review and meta-analysis. Sci Total Environ. 2020 Mar 1;706:135522.

17 Li MH, Fan LC, Mao B, Yang JW, Choi AMK, Cao WJ, et al. Short-term exposure to ambient fine particulate matter increases hospitalizations and mortality in COPD: a systematic review and meta-analysis. Chest. 2016 Feb; 149(2):447-58.

18 Hamra GB, Guha N, Cohen A, Laden F, Raaschou-Nielsen O, Samet JM, et al. Outdoor particulate matter exposure and lung cancer: a systematic review and meta-analysis. Environ Health Perspect. 2014;122(9):906-11.

19 Zheng XY, Ding H, Jiang LN, Chen SW, Zheng JP, Qiu M, et al. Association between air pollutants and asthma emergency room visits and hospital admissions in time series studies: a systematic review and meta-analysis. PLoS One. 2015;10(9):e0138146.

20 Yang BY, Fan S, Thiering E, Seissler J, Nowak $\mathrm{D}$, Dong $\mathrm{GH}$, et al. Ambient air pollution and diabetes: a systematic review and meta-analysis. Environ Res. 2020 Jan; 180:108817.

21 Shah AS, Langrish JP, Nair H, McAllister DA, Hunter AL, Donaldson K, et al. Global association of air pollution and heart failure: a systematic review and meta-analysis. Lancet. 2013 Sep 21;382(9897):1039-48.

22 Shah AS, Lee KK, McAllister DA, Hunter A, Nair H, Whiteley W, et al. Short term exposure to air pollution and stroke: systematic review and meta-analysis. BMJ. 2015;350: h1295.

23 Luo C, Zhu X, Yao C, Hou L, Zhang J, Cao J, et al. Short-term exposure to particulate air pollution and risk of myocardial infarction: a systematic review and meta-analysis. Environ Sci Pollut Res Int. 2015 Oct;22(19):14651-62.

24 Cullen B, Newby D, Lee D, Lyall DM, NevadoHolgado AJ, Evans JJ, et al. Cross-sectional and longitudinal analyses of outdoor air pollution exposure and cognitive function in UK Biobank. Sci Rep. 2018 Aug 14;8(1):12089.

25 Grande G, Ljungman PLS, Eneroth K, Bellander T, Rizzuto D. Association between cardiovascular disease and long-term exposure to air pollution with the risk of dementia. JAMA Neurol. 2020 Jul 1;77(7):801-9.

26 Kulick ER, Elkind MSV, Boehme AK, Joyce NR, Schupf N, Kaufman JD, et al. Long-term exposure to ambient air pollution, APOE- $\varepsilon 4$ status, and cognitive decline in a cohort of older adults in northern Manhattan. Environ Int. 2020 Mar; 136:105440.

27 Yuchi W, Sbihi H, Davies H, Tamburic L, Brauer M. Road proximity, air pollution, noise, green space and neurologic disease incidence: a population-based cohort study. Environ Health. 2020 Jan 21;19(1):8.

28 Weuve J, Puett RC, Schwartz J, Yanosky JD, Laden F, Grodstein F. Exposure to particulate air pollution and cognitive decline in older women. Arch Intern Med. 2012 Feb 13; 172(3):219-27.

29 Loop MS, Kent ST, Al-Hamdan MZ, Crosson WL, Estes SM, Estes MG Jr, et al. Fine particulate matter and incident cognitive impairment in the REasons for Geographic and Racial Differences in Stroke (REGARDS) cohort. PLoS One. 2013;8(9):e75001.

30 Tonne C, Elbaz A, Beevers S, Singh-Manoux A. Traffic-related air pollution in relation to cognitive function in older adults. Epidemiology. 2014 Sep;25(5):674-81.

31 Jung CR, Lin YT, Hwang BF. Ozone, particulate matter, and newly diagnosed Alzheimer's disease: a population-based cohort study in Taiwan. J Alzheimers Dis. 2015;44(2):573-84.

32 Cacciottolo M, Wang X, Driscoll I, Woodward N, Saffari A, Reyes J, et al. Particulate air pollutants, APOE alleles and their contributions to cognitive impairment in older women and to amyloidogenesis in experimental models. Transl Psychiatry. 2017 Jan 31;7(1):e1022.
33 Chen $\mathrm{H}$, Kwong JC, Copes R, Hystad P, van Donkelaar A, Tu K, et al. Exposure to ambient air pollution and the incidence of dementia: a population-based cohort study. Environ Int. 2017 Nov; 108:271-7.

34 Carey IM, Anderson HR, Atkinson RW, Beevers SD, Cook DG, Strachan DP, et al. Are noise and air pollution related to the incidence of dementia? A cohort study in London, England. BMJ Open. 2018;8(9): e022404.

35 Cleary EG, Cifuentes M, Grinstein G, Brugge D, Shea TB. Association of low-level ozone with cognitive decline in older adults. J Alzheimers Dis. 2018;61(1):67-78.

36 Oudin A, Segersson D, Adolfsson R, Forsberg $B$. Association between air pollution from residential wood burning and dementia incidence in a longitudinal study in Northern Sweden. PLoS One. 2018;13(6):e0198283.

37 Campbell M, Katikireddi SV, Sowden A, Thomson H. Lack of transparency in reporting narrative synthesis of quantitative data: a methodological assessment of systematic reviews. J Clin Epidemiol. 2019 Jan;105:1-9.

38 Ritchie K, Ritchie CW, Yaffe K, Skoog I, Scarmeas N. Is late-onset Alzheimer's disease really a disease of midlife? Alzheimers Dement. 2015;1(2):122-30.

39 Heusinkveld HJ, Wahle T, Campbell A, Westerink RHS, Tran L, Johnston H, et al. Neurodegenerative and neurological disorders by small inhaled particles. Neurotoxicology. 2016 Sep;56:94-106.

$40 \mathrm{Li} \mathrm{C}$, Martin RV, van Donkelaar A, Boys BL, Hammer MS, Xu JW, et al. Trends in chemical composition of global and regional population-weighted fine particulate matter estimated for 25 years. Environ Sci Technol. 2017 Oct 3;51(19):11185-95

41 Weagle CL, Snider G, Li C, van Donkelaar A, Philip S, Bissonnette P, et al. Global sources of fine particulate matter: interpretation of $\mathrm{PM}(2.5)$ chemical composition observed by SPARTAN using a Global Chemical Transport Model. Environ Sci Technol. 2018 Oct 16;52(20):11670-81.

42 Jones RR, Hoek G, Fisher JA, Hasheminassab $\mathrm{S}$, Wang D, Ward MH, et al. Land use regression models for ultrafine particles, fine particles, and black carbon in Southern California. Sci Total Environ. 2020 Jan 10;699:134234.

43 Killin LO, Starr JM, Shiue IJ, Russ TC. Environmental risk factors for dementia: a systematic review. BMC Geriatr. 2016 Oct 12;16(1): 175.

44 Power MC, Adar SD, Yanosky JD, Weuve J. Exposure to air pollution as a potential contributor to cognitive function, cognitive decline, brain imaging, and dementia: a systematic review of epidemiologic research. Neurotoxicology. 2016 Sep;56:235-53.

45 Fu P, Guo X, Cheung FMH, Yung KKL. The association between $\mathrm{PM}(2.5)$ exposure and neurological disorders: a systematic review and meta-analysis. Sci Total Environ. 2019 Mar 10;655:1240-8.
Evidence on PM2.5, Dementia, and Noncommunicable Disease
Neuroepidemiology 2021;55:253-265 DOI: $10.1159 / 000515394$ 